ÉGYPTE

monde arabe

\section{Égypte/Monde arabe}

$10 \mid 1992$

Partage de l'eau dans le monde arabe

\title{
Les enjeux pour Israël de la Conférence internationale sur l'eau au Proche-Orient
}

\section{Christian Chesnot}

\section{(2) OpenEdition}

12 Journals

Édition électronique

URL : https://journals.openedition.org/ema/1409

DOI : 10.4000/ema.1409

ISSN : 2090-7273

Éditeur

CEDEJ - Centre d'études et de documentation économiques juridiques et sociales

Édition imprimée

Date de publication : 30 juin 1992

Pagination : 51-62

ISSN : 1110-5097

Référence électronique

Christian Chesnot, « Les enjeux pour Israël de la Conférence internationale sur l'eau au ProcheOrient », Égypte/Monde arabe [En ligne], 10 | 1992, mis en ligne le 08 juillet 2008, consulté le 07 juillet 2022. URL : http://journals.openedition.org/ema/1409 ; DOI : https://doi.org/10.4000/ema.1409

Ce document a été généré automatiquement le 7 juillet 2022.

Tous droits réservés 


\title{
Les enjeux pour Israël de la Conférence internationale sur l'eau au Proche-Orient
}

\author{
Christian Chesnot
}

1 Le 12 mai 1992 s'ouvrait à Vienne, en présence de 130 experts et diplomates représentant 38 pays et organisations, la conférence internationale sur l'eau au ProcheOrient, ceci dans le cadre des négociations multilatérales israélo-arabes co-parrainées par les États-Unis et l'ex-Union soviétique. Cette conférence visait à jeter les bases d'une coopération dans un domaine constituant l'une des principales sources de tension dans la région et qui pourrait devenir, dans le futur, un des facteurs déterminants d'un éventuel conflit armé.

2 Malgré l'absence de deux pays directement concernés, le Liban et la Syrie (ces deux États ont refusé de participer aux discussions de Vienne, estimant que les négociations multilatérales ne peuvent être envisagées que lorsque des progrès tangibles auront été enregistrés dans les négociations de paix bilatérales) ${ }^{1}$, les multilatérales de Vienne ont constitué, selon les mots de M. Alexander Christiani, co-président autrichien de la conférence, «le commencement d'un processus à long terme».

Même si « l'atmosphère a été bonne », selon M. Christiani, des divergences profondes entre les participants sont apparues dès l'ouverture des travaux. Pour les Palestiniens, qui formaient une délégation commune avec les Jordaniens, la question de l'eau ne pourra être résolue que lorsqu'ils auront recouvré les droits dont ils ont été privés. Les délégués palestiniens ont par ailleurs fait état de statistiques selon lesquelles $6 \%$ des terres arables palestiniennes sont irriguées, contre 70\% des terres israéliennes, et plus de $50 \%$ des foyers palestiniens n'ont pas l'eau courante. Ce à quoi la délégation israélienne ${ }^{2}$ a répondu que «certaines questions doivent être traitées dans les négociations bilatérales mais qu'une série de problèmes techniques peuvent être discutés dans le forum multilatéral ». La conférence de vienne n'a donc débouché sur aucune décision concrète et s'est résumée à un « dialogue de sourds » entre Israéliens et Palestiniens, les premiers mettant délibérément l'accent sur l'aspect « technique » de 
la question, les seconds s'efforçant au contraire de souligner l'aspect «politique » du problème de l'eau dans le conflit israélo-arabe.

Au total, la réunion de Vienne a surtout permis aux différents protagonistes d'exprimer leur point de vue. Quatre groupes de travail ont été mis en place, relatifs à l'approfondissement des informations disponibles sur l'inventaire et les besoins en eau de chaque État de la région, l'amélioration des réserves aquifères, la gestion des ressources hydrauliques et la coopération.

5 D'ores et déjà, il apparaît que le problème du partage de l'eau au Proche-Orient sera des plus délicats à résoudre tant ses implications - stratégiques, politiques, économiques, agricoles, démographiques et environnementales - sont multiples et complexes.

6 Comme la plupart des États de la région, Israël redoute pour les années à venir une grave pénurie d'eau, ses ressources aquifères étant limitées et déjà surexploitées tandis que sa consommation ne cesse de croître.

7 Grâce à ses victoires militaires de 1948 et 1967, l'État hébreu a pu s'assurer le contrôle des ressources hydrauliques du Golan syrien et des affluents du Jourdain ainsi que de la quasi-totalité des sources et nappes aquifères de la Cisjordanie et de la bande de Gaza. En outre, l'invasion du Sud-Liban en 1978 lui a permis de prendre le contrôle des fleuves Litani et Wazzani. Selon le journal AI-Hayat ${ }^{3}$, Israël a ainsi augmenté son potentiel en eau de 1.200 millions de mètres cubes à la suite de la guerre de 1967 et de l'invasion du Liban-sud.

8 La totalité des ressources hydrauliques israéliennes, dont $85 \%$ sont situées dans le nord du pays, avoisine les 1.650 millions de mètres cubes et se répartit comme suit ${ }^{4}$ :

- nappes souterraines : 950 millions de $\mathrm{m}^{3}$;

- Jourdain et lac de Tibériade : 600 millions de $\mathrm{m}^{3}$;

- eaux de ruissellement : 60-100 millions de $\mathrm{m}^{3}$.

9 Par ailleurs, le niveau des précipitations qui servent à la fois à l'irrigation directe et au réapprovisionnement de la nappe phréatique fournissant l'eau des puits et des sources, varie entre 1000 millimètres par an dans le nord (Safad) et 39 millimètres par an dans le sud (Eilat) $)^{5}$.

La totalité des ressources hydrauliques de la Cisjordanie s'élève à environ 850 millions de mètres cubes et se répartit comme suit ${ }^{6}$ :

- nappes souterraines : 600 millions de $\mathrm{m}^{3}$;

- Jourdain et cours d'eau : 250 millions de $\mathrm{m}^{3}$.

11 Les ressources en eau de la bande de Gaza sont quant à elles évaluées à 50-80 millions de mètres cubes. On estime d'autre part que $90 \%$ de l'eau extraite de Cisjordanie servent à la consommation d'Israël alors que les Palestiniens ne bénéficient que de $10 \%$ de ce volume.

12 Actuellement, l'État hébreu exploite $98 \%$ de ses ressources hydrauliques et consomme 2.145 millions de mètres cubes d'eau ${ }^{7}$. D'où son intérêt évident pour des sources situées hors de ses frontières.

La consommation d'eau en Israël se répartit comme suit ${ }^{8}$ :

- agriculture : 75 à $80 \%$;

- utilisation domestique : 15 à $18 \%$;

• industrie : 4 à $6 \%$.

Selon A. Mansour ${ }^{9}$, 
« le besoin d'Israël en eau constitue un des facteurs fondamentaux déterminant la politique israélienne dans les territoires occupés. Les restrictions imposées à ces territoires sur le plan de leur développement économique ont pour objet, entre autres, de réduire la consommation d'eau de la population palestinienne. La question de l'eau est, plus que toute autre, liée à un problème politique. Le contrôle politique et militaire de la Cisjordanie permet en fait à Israël de s'assurer le contrôle des nappes aquifères situées sur le versant occidental de la Cisjordanie. Tout retrait israélien de cette région lui ferait perdre le contrôle de ces ressources et causerait ainsi de graves préjudices à l'économie israélienne et plus particulièrement à son agriculture. "

15 Il est clair que dans les prochaines années, Israël va devoir gérer un écart croissant entre la quantité d'eau disponible et sa consommation nationale. D'ici l'an 2000, l'État hébreu pourrait souffrir d'un déficit permanent en eau de l'ordre de $30 \%$. L'évolution de la consommation d'eau en Israël sera tributaire de trois facteurs :

- l'évolution du rythme de l'immigration des juifs soviétiques ;

- l'évolution de la politique de colonisation des territoires occupés ;

- l'évolution du secteur agricole israélien.

16 L'un des défis majeurs que doit relever l'État hébreu est sans conteste celui de sa démographie. En 23 ans, la population israélienne a doublé, passant de 2,6 millions d'habitants en 1967 à 5.2 millions en 1990. De plus, la dislocation de l'Union Soviétique a récemment permis l'immigration de nombreux juifs soviétiques vers Israël.

17 Ainsi, depuis 1989, 400.000 juifs soviétiques s'y sont installés et, selon les responsables israéliens. 1.2 million de juifs répartis dans les 15 anciennes républiques de l'ex-URSS désirent les suivre. Même si le rythme des arrivées s'est quelque peu tassé (en 1991, selon les chiffres officiels israéliens, seulement 140.000 juifs soviétiques ont immigré en Israël alors que près de 400.000 étaient attendus), cet accroissement démographique soudain et massif pèsera sur la consommation d'eau : avec cet afflux, Israël aura besoin de 268 millions de $\mathrm{m}^{3}$ d'eau supplémentaires dans les trois prochaines années ${ }^{10}$.

18 Autre élément à prendre en compte : l'intensification, depuis la conférence de paix de Madrid en octobre 1991, de la politique d'implantations dans les territoires occupés (même si, au mois de juillet 1992, le nouveau gouvernement travailliste dirigé par Yitzhak Rabin a décidé de geler partiellement et temporairement tes implantations en Cisjordanie et dans la Bande de Gaza). Selon un rapport du ministère américain des Affaires étrangères, les implantations dans les territoires conquis par l'État hébreu en 1967 se répartissent comme suit ${ }^{11}$ :

- Cisjordanie : 90.000 colons installés dans 150 implantations,

- Bande de Gaza : 3.000 colons installés dans 15 implantations,

- Golan syrien : 12.000 colons installés dans 30 implantations.

19 Outre leur aspect politique et idéologique, les implantations Juives sont très dispendieuses en matière d'utilisation des ressources aquifères. Ainsi, un Israélien consomme 375 mètres cubes d'eau par an, un Palestinien de 107 à 156 et un colon de 640 à 1.480. En effet, afin de développer sa politique de colonisation, Israël subventionne très fortement l'eau utilisée par les colons, notamment par le biais de l'Organisation sioniste mondiale. Un colon paie donc 15 agorot le mètre cube d'eau destiné à l'agriculture et 23 agorot le mètre cube d'eau à usage domestique. Quant au consommateur palestinien, il doit payer 70 agorot l'administration israélienne pour chaque mètre cube d'eau consommé, quelle que soit son utilisation ${ }^{12}$. 
20 Toutes les implantations sont alimentées en eau, condition sine qua non de leur développement. Mais la manifestation la plus révélatrice de la surconsommation d'eau dans les colonies de peuplement israéliennes est encore la présence de nombreuses piscines $^{13}$. Bien plus, selon J. D. Oillman ${ }^{14}$, «Israël impose maints obstacles légaux qui perpétuent une inégalité de traitement entre Juifs et Palestiniens. [...] Des restrictions sont imposées en matières de forage et d'utilisation des puits. [...] Avec la législation israélienne actuelle, les nouveaux permis de forage ne sont qu'exceptionnellement accordés. Par ailleurs, la profondeur des puits palestiniens ne dépasse généralement pas les 100 mètres alors que les puits des colons atteignent couramment 300 à 500 mètres. Les autorités israéliennes ont également autorisé ces derniers à procéder à de nouveaux forages près de l'emplacement des puits palestiniens déjà existants. »

21 Cette politique discriminatoire, qui répond à des considérations politiques et économiques, pénalise fortement l'agriculture palestinienne. Ainsi par exemple dans la bande de Gaza, les producteurs de citrons, principale culture de cette région, sont particulièrement touchés par le rationnement imposé. En 1991, tous les fermiers de Gaza ont vu leur quota d'eau arbitrairement réduit de $10 \%$ par l'administration israélienne. En 1992, la quantité d'eau utilisable a de nouveau été diminuée de 10\%. Or, la culture du citron exige 1.000 mètres cubes d'eau par dunam et par an: en 1990, les producteurs de citrons ont reçu une allocation de $1.000 \mathrm{~m}^{3}$, qui a été réduite à $900 \mathrm{~m}^{3} \mathrm{en}$ 1991, et à $810 \mathrm{~m}^{3}$ pour 1992. Les producteurs palestiniens qui ne respectent pas ces quotas sont passibles d'amendes. La mise en place de cet arsenal de contraintes destinées à limiter l'accès des Palestiniens à l'eau répond à deux objectifs; assurer te développement des colonies de peuplement et protéger l'agriculture israélienne de la concurrence palestinienne.

22 Car le troisième facteur qui conditionnera la consommation en eau d'Israël, outre l'immigration de juifs et la colonisation des territoires occupés, concerne l'évolution du secteur agricole israélien.

Dans l'idéologie sioniste, l'eau et le développement de l'agriculture occupent une place prépondérante. Le fameux slogan " faire fleurir le désert », brandi par les fondateurs de l'État d'Israël est profondément ancré dans la mentalité israélienne. D'où un développement rapide du secteur agricole qui tient aussi pour une bonne part à des impératifs de sécurité alimentaire. Toutefois, ce secteur fortement capitalistique et hautement mécanisé représente $5 \%$ du produit national brut israélien mais absorbe plus de $70 \%$ de l'eau disponible (voir plus haut). Contrairement à une idée largement répandue, l'agriculture israélienne n'est pas économe en eau. C'est pourquoi, depuis plusieurs années déjà, des voix s'élèvent en Israël pour contester la priorité donnée à l'agriculture au moment où l'eau devient précieuse parce que rare. Certains préconisent de développer des industries et des secteurs de pointe à haute valeur ajoutée plutôt que de continuer à investir dans un secteur agricole lourdement subventionné par l'État et gros consommateur d'eau.

Si l'on en croit le Jerusalem Post ${ }^{15}$, «le développement de l'agriculture et les subventions accordées aux fermiers ont toujours été considérés comme des " vaches sacrées », l'une des conditions sine qua non du sionisme. Autant des slogans comme la 'bonification de la terre" ou encore "un dunam par ici, un dunam par là ", étaient essentiels et constructifs sous le mandat britannique, autant aujourd'hui, ils ne conviennent plus à un État souverain. [...] Pour le contribuable, subventionner des productions agricoles vendues moins chères que le prix de l'eau qui a servi à les irriguer, n'est pas seulement 
une absurdité économique mais aussi un véritable suicide. » De même, pour Arie Issar ${ }^{16}$, professeur d'hydrologie à l'Institut de recherche sur le désert israélien, « l'idée de faire fleurir le désert a constitué l'un des piliers fondamentaux du mouvement sioniste, mais ce rêve est devenu perverti. »

Par ailleurs, le développement agricole a entrainé des répercussions négatives sur l'environnement et la qualité de certaines sources d'eau, polluées par l'emploi excessif et généralisé de fertilisants et de pesticides : Israël est aujourd'hui le pays au monde qui en utilise le plus ${ }^{17}$.

Confronté à une consommation en expansion et qui dépendra de l'évolution des trois facteurs cités plus haut, et à défaut de ressources aquifères insuffisantes pour satisfaire ces nouveaux besoins, il sera difficile à Israël d'abandonner le contrôle de deux zones riches en eau : le Sud-Liban et le Golan syrien. Deux régions où les intérêts relevant de la «sécurité militaire " israélienne coïncident parfaitement avec les impératifs ayant trait à la " sécurité hydraulique » de l'État hébreu. Depuis longtemps, les eaux du SudLiban et en particulier de son principal fleuve, le Litani - 300 millions de $\mathrm{m}^{318}$ intéressent les responsables israéliens. Dès 1916, le mouvement sioniste voulait faire du fleuve Litani la frontière nord du futur État juif :

« Les frontières [du foyer national juif] ne sauraient être tracées exclusivement sur la base des limites historiques [bibliques] [...]. Nos prétentions vers le nord sont impérativement dictées par les nécessités de la vie économique moderne [...]. Tout l'avenir économique de la Palestine dépend de son approvisionnement en eau pour l'irrigation pour la production d'électricité; et l'alimentation en eau doit essentiellement provenir des pentes du mont Hermon, des sources du Jourdain et $\mathrm{du}$ fleuve Litani [...]. Nous considérons qu'il est essentiel que la frontière nord de la Palestine englobe la vallée du Litani sur une distance de près de 25 miles [40,5 km environ] en amont du coude, ainsi que les flancs ouest et sud du mont Hermon ${ }^{19}$ ».

Depuis l'invasion du Sud-Liban en 1978 et l'opération « Paix en Galilée » de juin 1982, le Liban a régulièrement accusé Israël de détourner les eaux du Litani. Toutefois, il n'en existe aucune preuve formelle; le ministre libanais des Affaires étrangères, Farès Boueiz, l'a d'ailleurs reconnu; "Nous n'avons pas d'informations précises, fondées et véritables qui indiqueraient qu'il y a vol de l'eau du côté israélien, jusqu'à présent et de façon directe, à partir du Liban-sud. $»^{20}$

Israël justifie la poursuite de son occupation du Liban-sud par des impératifs de sécurité: la présence de l'armée israélienne dans cette région serait destinée à empêcher le Hezbollah et la résistance libanaise d'attaquer la « zone de sécurité » et le territoire israélien, toutes opérations qualifiées de «terroristes» par l'État hébreu. Ainsi a-t-il toujours affirmé qu'il lui serait impossible de se retirer du Liban-sud tant que demeurerait la menace d'éventuelles attaques venant de cette région et que les troupes syriennes n'auraient pas évacué le territoire libanais. L'État hébreu a aussi fait savoir-il est intéressant de le noter - qu'il ne se retirerait pas du Liban-sud sans avoir obtenu des facilités d'accès à l'eau du Litani ${ }^{21}$.

Bref, les questions de sécurité militaire et de sécurité hydraulique sont ici intimement liées. C'est aussi le cas pour le plateau du Golan syrien, conquis par Israël en 1967 et annexé en décembre 1981. Cette région, délimitée au nord par le mont Hermon (2.800 mètres), à l'ouest par la dépression du Houleh, du Jourdain et du lac de Tibériade (- 212 mètres), au sud par la vallée du Yarmouk qui marque la frontière syro-jordanienne et à l'est par le Rakkad, l'affluent du Yarmouk, est riche, tout comme le Liban-sud, en 
ressources hydrauliques : le Jourdain y prend une de ses sources et le Banias alimente le lac de Tibériade (330 millions de mètres cubes).

Militairement, le plateau du Golan constitue une position stratégique de tout premier ordre. Les Romains, pendant l'Antiquité, y avaient même installé des garnisons pour protéger les réserves d'eau. Les Syriens en feront une place-forte, utilisant son relief accidenté pour installer lignes d'artillerie et bunkers. Comme dans le cas du Liban-sud, Israël a justifié l'occupation puis l'annexion du Golan pour des raisons de sécurité : il s'agit d'éviter que les kibboutz et les localités Juives de Galilée ne soient à la portée des canons syriens. Les Israéliens présentent donc le Golan comme une région vitale pour leur "sécurité nationale ». Mais là encore, comme pour le Liban, intérêt militaire et intérêt hydraulique se mêlent étroitement: le Golan et ses 200 millions de $\mathrm{m}^{3}$ d'eau constituent une source d'approvisionnement non négligeable pour l'État hébreu.

Conscients de l'intérêt que représente le contrôle de certaines sources d'eau pour son approvisionnement (Cisjordanie, bande de Gaza, plateau du Golan, Liban-sud), les responsables israéliens ont adopté, lors des négociations de paix, une stratégie visant à dépouiller la question de l'eau de son aspect éminemment politique pour faire porter le débat sur le côté « technique » du problème.

En effet, afin de prouver sa bonne volonté pour résoudre cette question, Israël se dit prêt à coopérer dans ce domaine avec les différents États de la région, et notamment à faire partager son savoir-faire et sa technologie en matière d'irrigation des zones désertiques, de dessalement de l'eau de mer ou encore de traitement des eaux saumâtres: la mise en commun des technologies et la mise sur pied de projets de coopération, estiment les Israéliens, peuvent impulser une dynamique au processus de paix en créant un climat de confiance.

Le Professeur Dan Zasiavski, membre de la délégation israélienne à la conférence de Vienne $^{22}$, donne sa vision du déroulement des négociations :

«Israël propose de résoudre la question de l'eau par des moyens scientifiques et d'exclure toute attaque tendant à accuser Israël de capter l'eau des Arabes, car Israël pense que l'échange d'accusations entrave la paix [...]. Je crois que dans une première phase, il faudrait nous concentrer sur les sources d'eau non utilisées, et il y en a beaucoup; sans entrer dans les délais. On peut citer l'eau que la Jordanie n'utilise pas, tout le long de la frontière avec Israël ».

Mais dans tous les cas, poursuit le professeur Zasiavski, «Israël propose la paix contre la paix et rien d'autre $»^{23}$. Une formule ambiguë qui traduit les réticences israéliennes concernant d'éventuels arrangements territoriaux qui, de facto, auraient des conséquences sur les ressources hydrauliques de l'État hébreu, comme le rappelle Magdi Sobh du Centre d'études stratégiques d'Al-Ahrâm, «Israël, qui a besoin de l'eau des territoires occupés pour sa consommation, souhaite obtenir des garanties de la part des Palestiniens, afin que ceux-ci ne contrôlent pas toute l'eau de la Cisjordanie. Toutefois, si Israël se retirait de Cisjordanie et de la bande de Gaza, sa quantité d'eau disponible diminuerait fortement mais en revanche il y aurait de réelles possibilités de coopération. $»^{24}$

Selon le professeur Georges Gruen de l'université américaine de Columbia, les Palestiniens, au cours des négociation; de paix, vont s'efforcer de prendre en charge "les eaux palestiniennes", tandis que les Israéliens vont utiliser l'argument selon lequel ils exploitaient ces sources d'eau bien avant la guerre de 1967, et revendiquer un droit historique fondé sur la tradition de l'usage de ces sources ${ }^{25}$. 

l'eau dans la région passe d'abord par la résolution des questions politiques et, au premier chef d'entre elles, la question de la terre et du retour aux frontières d'avant 1967. faire face à son déficit en d'eau? On peut en citer quatre, qui ne s'excluent pas et peuvent même se combiner :

- importer de l'eau de l'extérieur,

- développer sur une grande échelle les techniques de dessalement de l'eau de mer,

- restructurer son économie dans le sens d'une réduction du secteur agricole,

- maintenir par la force, le plus longtemps possible, le statu quo territorial.

\section{Importer de l'eau de l'extérieur}

Actuellement, Israël importe de l'eau de Turquie, une décision prise après des années de sécheresse entre 1987 et $1991^{26}$. Israël serait en train d'étudier la possibilité de faire venir l'eau de Bulgarie, de Yougoslavie, d'Italie, de Roumanie et d'Égypte ${ }^{27}$. Ce dernier pays a cependant récemment annoncé que les eaux du Nil étaient exclues des discussions de Vienne et qu'il n'était pas question qu'Israël en importe.

Cette option, malgré son coût en transport, présente l'avantage pour Israël de préserver une partie de ses ressources hydrauliques, l'inconvénient majeur étant de se mettre en situation de dépendance vis-à-vis de pays qui pourraient, selon les circonstances, exercer un « chantage » à l'eau. La Turquie et son projet «d'aqueduc de la paix » pourrait desservir l'État hébreu, mais pour le moment, rien n'a encore été décidé.

\section{Développer à grande échelle les usines de dessalement de l'eau de mer}

40 Ce procédé est déjà très largement utilisé dans les pays du Golfe qui tirent $70 \%$ de leurs besoins en eau potable d'une quarantaine d'usines de dessalement. Mais pour construire une telle infrastructure, ces pays disposaient d'une capacité financière considérable tirée de leurs revenus pétroliers.

41 Selon une étude réalisée par le ministère néerlandais des Affaires étrangères sur les ressources aquifères de la bande de $\mathrm{Gaza}^{28}$, le coût de cette technique revient à 1.5/2 dollars par mètre cube d'eau dessalée. Israël utilise déjà le dessalement mais à une faible échelle. Généraliser cette technologie exigerait d'importants investissements financiers.

\section{Restructurer l'économie israélienne dans le sens d'une réduction du secteur agricole}

Comme nous l'avons vu plus haut, l'agriculture israélienne est très dispendieuse en eau et a constitué l'un des socles principaux sur lequel l'État hébreu s'est développé depuis sa fondation en 1948. Mais diminuer les investissements dans ce secteur, c'est aborder

Égypte/Monde arabe, 10 | 1992 
la question de la sécurité alimentaire d'Israël. Par ailleurs, il faudra compter avec le poids du puissant lobby des agriculteurs, habitués à ne pas payer l'eau à sa juste valeur $^{29}$. Toutefois, des mesures d'économie ont été déjà introduites, comme la réduction de certaines cultures exigeant des fortes quantités d'eau, notamment le coton. Bref, les responsables israéliens devront repenser l'empiétement, la place et le rôle de l'agriculture dans l'économie. Mais il est clair que c'est dans ce secteur que les plus grandes économies d'eau pourront être réalisées et peser de façon significative sur la consommation nationale.

\title{
Maintenir par la force le statu quo territorial le plus longtemps possible
}

Pour le Dr Saïd Rouchdy, géologue égyptien ${ }^{30}$,

\begin{abstract}
« à long terme, Israël n'a pratiquement pas le choix, si ce n'est d'établir des relations pacifiques avec ses voisins. Il peut ou bien réorganiser son économie en réduisant le secteur agricole, ou bien militariser un peu plus la question de l'eau et s'engager alors dans une nouvelle guerre pour l'eau. Selon moi, il sera très difficile à Israël de choisir entre ces deux possibilités. La première option serait impossible en l'absence de paix et la seconde particulièrement difficile à mettre en œuvre avec les nouveaux paramètres introduits par le nouvel ordre international ».
\end{abstract}

Cette dernière hypothèse ne peut constituer une solution durable, notamment parce que l'État hébreu devrait alors consacrer une part de plus en plus importante de son budget à la défense, qui se taille déjà actuellement la part du lion dans les dépenses de l'État hébreu. À l'inverse, dans le cas d'un règlement politique au Proche-Orient, Israël pourrait alors maximiser les effets positifs d'une coopération régionale, le règlement de la question passant alors par un partage négocié et équitable des ressources en eau, et plus particulièrement du Jourdain.

L'issue des multilatérales sur l'eau revêt une importance cruciale pour Israël. Néanmoins, devant l'évolution actuelle du processus de paix, la conclusion d'un accord sur l'eau au Proche-Orient prendra du temps ${ }^{31}$. Car les pays arabes subordonnent toute coopération régionale, dans quelque domaine que ce soit, à de réels progrès dans les bilatérales. Pourtant, étant donné l'état des réserves hydrauliques du Proche-Orient et les besoins croissants de chaque État, les gouvernements devront trouver un minimum de terrain d'entente s'ils ne veulent pas que la " compétition pour l'eau » ne dégénère en conflit armé à plus ou moins longue échéance. Pour le moment, c'est Israël, par son occupation du Golan, de la Cisjordanie, de la bande de Gaza et du Liban-sud, qui détient les clés d'un éventuel règlement de cette question.

\section{NOTES}

1. Trois pays importants du Moyen-Orient n'ont pas été invités à la conférence de Vienne ; il s'agit de la Libye, de l'Iran et de l'Irak. 
2. Outre le premier round des négociations multilatérales, qui se sont déroulées à Vienne en mai, Israël a participé aux multilatérales sur le désarmement (Washington) le sur la protection de l'environnement (Tokyo), mais n'était pas présent aux multilatérales sur les réfugiés (Ottawa) et sur la coopération économique (Bruxelles) du fait de la présence de Palestiniens de l'intérieur.

3. Al-Hayat, 26 janvier 1992.

4. YAKOBOWITZ (M.) et PRUSHANKY (Y.), 1987, The water in Israël. Israël information Center, Jérusalem.

5. KAHHALEH (S,), 1981, The water problem in Israël and its repercussions on the Arab-Israëli conflict, Institute for Palestine Studies, Beyrouth.

6. cf. note 4 .

7. Al-Hayat. 26 Janvier 1992.

8. cf. note (5).

9. MANSOUR (A.), 1983, Palestine; une économie de résistance enCisjordanie et à Gaza, l'Harmattan.

10. Al-Ahram Weekly, 20 février 1992.

11. Al-Hayat, 9 janvier 1992. NB : cette étude ne tient pas compte des quelque 150,000 Israéliens qui résident à Jérusalem-Est occupée et annexée.

12. BENVENISTI (M.) et KHAYAT (S.), 1988, « The West Bank and Gaza Atlas », The Jerusalem Post.

13. Entretien de l'auteur avec des Palestiniens de Gaza (mars 1992).

14. DILLMAN (J. D.), 1989, «Water rights in the Occupied Territories ", Journal of Palestine Studies.

15. Éditorial du Jérusalem Post intitulé « Que faire à propos de l'eau? », 4 janvier 1991.

16. Cité par le journal londonien The Guardian, 6 décembre 1991.

17. Revue Tanmiya, numéro 25, décembre 1991.

18. Chiffre cité dans Al-Hayat, 7 mars 1992.

19. Lettre de Chaim Weizmann, un des leaders du mouvement sioniste, adressée en 1919 au Premier ministre britannique de l'époque Lloyd George. Cité dans « Water rights in the Occupied Territories ", cf. note 14.

20. Interview de Farès Boueiz publiée dans Al-Wasat, 27 avril 1992.

21. Al-Hayat, 27 Janvier 1992.

22. Interview du professeur Zaslavski publiée dans Al-Ahram le 2 mars 1992 sous le titre : «Les sources d'eau arabes sont nombreuses et nous pourrons les utiliser ensemble ».

23. cf. note 22 .

24. Entretien avec l'auteur.

25. GRUEN (G.), La Crise de l'eau : le prochain conflit du Moyen-Orient? (Cité dans Al-Ahram Weekly, 19 mars 1992).

26. Al-Ahram Weekly, 20 février 1992.

27. The Jerusalem Post, 31 décembre 1990.

28. BRUINS (H. J.), TUINHOF (A.) et KELLER (R.), septembre 1991, Water in the Gaza strip, ministère des Affaires étrangères des Pays-Bas.

29. The Jerusalem Post, 3 janvier 1991.

30. Al-Ahram Weekly, 20 février 1992.

31. À l'heure où nous mettons sous presse (mi-septembre 1992), les Jordaniens et les Israéliens se sont mis d'accord, selon une source jordanienne, pour inscrire la question de l'eau à l'ordre du jour de leurs négociations bilatérales. 
INDEX

Mots-clés : eau, Israël, stratégie 